\title{
A Design Framework for Predictive Engine Control
}

\author{
X. Wang, H. Waschl, D. Alberer ${ }^{*}$ and L. del Re \\ Institute for Design and Control of Mechatronical Systems, Johannes Kepler University Linz, 4040 Linz - Austria \\ e-mail: xiaoming.wang@jku.at - harald.waschl@jku.at - daniel.alberer@jku.at - luigi.delre@jku.at \\ * Corresponding author
}

\begin{abstract}
Résumé - Un cadre de conception pour la commande prédictive de moteurs - La commande prédictive par modèle (Model Predictive Control; $M P C$ ) a été proposée plusieurs fois dans l'automatique pour l'automobile, avec des résultats prometteurs, principalement à partir d'une approche MPC linéaire. Toutefois, comme la plupart des systèmes automobiles sont non linéaires, la commande prédictive non linéaire (Nonlinear MPC; $N M P C$ ) pourrait représenter une option intéressante. Malheureusement, la conception d'une commande optimale à partir d'un modèle non linéaire générique conduit généralement à un problème non convexe complexe. Dans ce contexte, cet article présente deux schémas différents pour prendre en compte la non linéarité du système en vue de la conception de la commande. En premier lieu, une méthode MPC multilinéaire est présentée sur la base d'une segmentation du système et, en second lieu, une conception de système de commande basée sur une identification de système non linéaire utilisant une structure quasi linéaire à paramètres variants (Linear Parameter Varying; LPV) est proposée ; celle-ci est alors utilisée dans un cadre de conception de NMPC. Cet article présente ces approches et leur application à un système bien connu, le module d'air (air path) d'un moteur Diesel.
\end{abstract}

\begin{abstract}
A Design Framework for Predictive Engine Control - Model Predictive Control (MPC) has been proposed several times for automotive control, with promising results, mostly based on a linear MPC approach. However, as most automotive systems are nonlinear, Nonlinear MPC (NMPC) would be an interesting option. Unfortunately, an optimal control design with a generic nonlinear model usually leads to a complex, non convex problem. Against this background, this paper presents two different schemes to take into account the system nonlinearity in the control design. First, a multi-linear MPC method is shown based on the segmentation of the system and then a control system design based on a nonlinear system identification using a quasi Linear Parameter Varying (LPV) structure is proposed, which is then used in a NMPC design framework. This paper presents the approaches and the application to a well studied system, the air path of a Diesel engine.
\end{abstract}




\section{INTRODUCTION}

The continuously tightened emission limits oblige the automotive industry to improve their combustion engines. This can either be done by changes in the hardware (e.g. by application of catalysts or advanced injection system etc.) or by modifications in the software, especially the control strategy for fuel injection and the air path system. Standard production engine controls are still developed in a very heuristic way and reach a huge and fault-prone complexity. Even though this is widely recognized and model based control is almost unanimously advocated as the solution of choice, the enormous legacy of decades of heuristic solutions makes the transition slow and uncertain. Against this background this paper suggests a systematic approach for the identification of control models and their application in the framework of Model Predictive Control (MPC) to the air path of a production Diesel engine, whereas the focus clearly lies on the design method.

From a control point of view the air path of a Diesel engine is a very challenging system, namely a highly coupled nonlinear MIMO system with constraints and a limited feasible working range. Figure 1, for instance, depicts stationary measurements of fresh air mass flow $(M A F)$ and boost pressure $(M A P)$ for changing air path actuator values. As it can be seen, the DC-gains are not constant and even change their $\operatorname{sign}^{1}$, which obviously cannot be captured by a linear model.

Many works present the application of model based MIMO control to the Diesel engine air path. Stefanopoulou et al. (2000) for instance show a combination of a nonlinear feedforward and a gain scheduled MIMO controller for the control of the burnt mass fraction of the intake and the air fuel ratio or van Nieuwstadt et al. (2000) compared several controller approaches. Robust nonlinear air path control was applied in Jankovic and Kolmanovsky (1998) and more in detail in Jankovic et al. (2000), where input-output linearization was used for the generation of a control Lyapunov function and a controller with a guaranteed robustness property was determined. Flatness based polynomial control is shown in Ayadi et al. (2004), and Jung (2003) as well as Wei (2006) show approaches of robust Linear Parameter Varying (LPV) control for the air path.

Due to its capability of handling constraints on manipulated variables and system states and the explicit minimization of a cost function, the use of Model Predictive Control for engine control has recently attracted much interest. Rückert et al. (2004) implemented a gain scheduled Generalized Predictive Control (GPC) strategy for a truck Diesel engine and

\footnotetext{
${ }^{1}$ Due to the high exhaust back pressure at minimum turbine guide vane opening $(V G T \rightarrow 100 \%)$ the EGR flow is increased and consequently the fresh air mass flow is reduced (Fig. 1a). Moreover, at high EGR flow (e.g. 70\% EGR-valve position) the turbine flow and thus the turbine power is reduced, which causes a drop of boost pressure (Fig. 1b). All this strongly depends on the engine design.
}
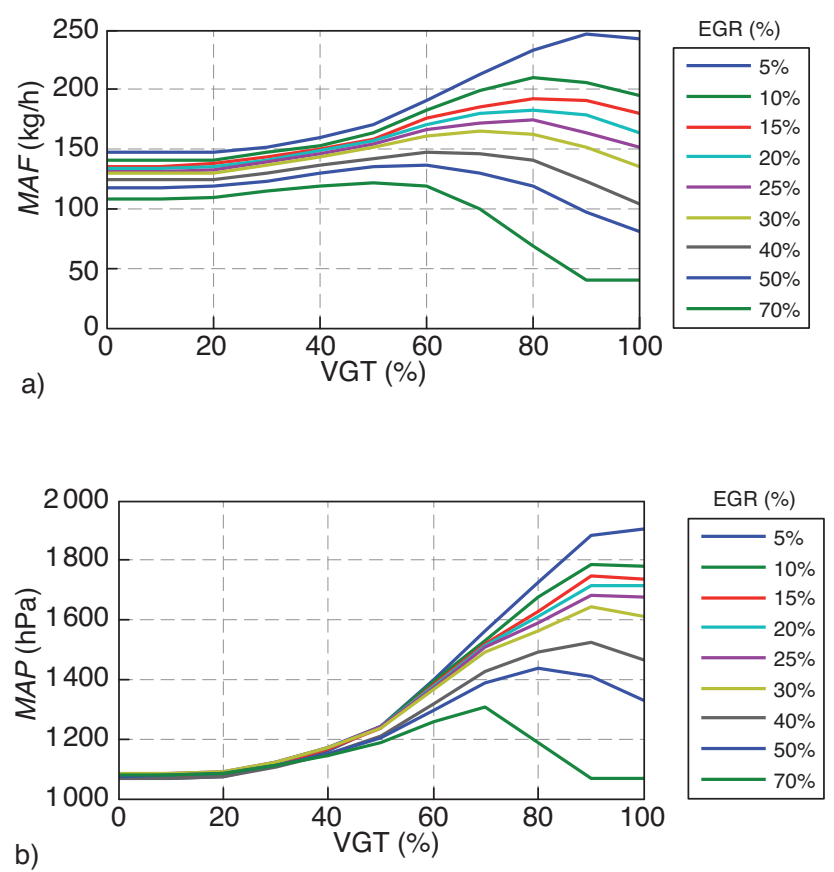

Figure 1

Stationary measurements of fresh air mass flow a) and boost pressure b) at different air path actuator positions.

Dynamic Matrix Control (DMC) and GPC strategies were developed in Garcia-Ortiz (2004) and Salcedo et al. (2004). To cope with the nonlinearity of the system, different approaches exist, e.g. Ortner and del Re (2007) and Langthaler (2007) introduce a segmentation of the working range and design separate linear MPCs for every segment. The logic alternative is to design a single Nonlinear MPC (NMPC) for the whole engine operating range, e.g. Herceg et al. (2006) present a simulation study for the application of NMPC to a Diesel engine air path.

Unfortunately, very few attempts have been done in this way in practice, because of several reasons, the two most important ones being the lack of models required to compute the solution and the limited CPU power, in particular in the case of a production Engine Control Unit (ECU). Furthermore, efficient solvers are required which are able to compute the solution of the Quadratic Programs (QPs) which are arising in Model Predictive Control. In Ferreau et al. (2006, 2008) an online active set strategy for QPs is presented, which has already been successfully applied to air path control at an engine test-bed (Ferreau et al., 2007).

The actual paper builds on this experience and compares two different methods of how to take into account the system nonlinearities in the control design. First, the above mentioned approach of the segmentation is discussed. To this end, multiple linear models were identified for restricted 
operating regimes and several linear MPCs were applied to the engine - according to the actual system state only one linear MPC had the control authority. With the second method a generalization is presented using a single nonlinear model, albeit one which is easy to linearize at each operating point. This is done combining an LPV identification technique with a simple formulation of NMPC based on the recursive use of the above mentioned QP solver with a guaranteed computation time. The paper explains the methods and presents their use for the very well known example of the fresh air mass flow $(M A F)$ and boost pressure $(M A P)$ control of a Diesel engine air path.

\section{THE DESIGN FRAMEWORK}

As well known, MPC uses model information to predict the states of the system over a finite horizon and to compute accordingly the optimal trajectory (see Fig. 2). The problem typically solved by MPC in the case of a linear system with constraints on the inputs and the states can be resumed as follows:

$$
\min _{u_{k} \in u} \frac{1}{2} \sum_{k=0}^{N P H}\left[\left(y_{k}-y_{r e f}\right)^{T} Q\left(y_{k}-y_{r e f}\right)+\Delta u_{k}^{T} R \Delta u_{k}\right]
$$

s.t.

$$
\begin{array}{ll}
\Delta u_{k}=u_{k}-u_{k-1} & \\
x_{k+1}=A_{k} x_{k}+B_{k} u_{k}+Z v & \\
y_{k}=C_{k} x_{k} & \\
\underline{x} \leq x_{k} \leq \bar{x} & \forall k=0 \ldots N P H \\
\underline{u} \leq u_{k} \leq \bar{u} & \forall k=N C H . . N P H \\
\Delta u_{k}=0 &
\end{array}
$$

The solution of this optimization problem is the future control sequence $u^{*}=\left[u_{k}, u_{k+1}, \ldots, u_{k+N C H-1}\right]$, where only the first value will be applied and the optimization is repeated at the next sampling instant. $N C H$ and $N P H$ are called control and prediction horizon. The coefficients $Q$ and $R$ are positive definite weighting matrices to penalize the tracking error and the control effort respectively. $y_{\text {ref }}$ is the output reference trajectory, $x$ denotes the vector of system states, $u$ the vector of manipulated variables and $v$ forms the vector of measured disturbances. $\underline{x}, \bar{x}$ and $\underline{u}, \bar{u}$ represent lower and upper bounds on the states and the manipulated variables, which means that constraints can be taken into account explicitly in the controller design.

Inserting the linear model $x_{k+1}=A_{k} x_{k}+B_{k} u_{k},+Z_{v}$, $y_{k}=C_{k} x_{k}$ into the optimization task (1) yields a so called Quadratic Program (QP), in which the sequence $u^{*}$ can be separated from the prediction part and this convex problem can be solved using a standard QP solver. Nonlinear MPC

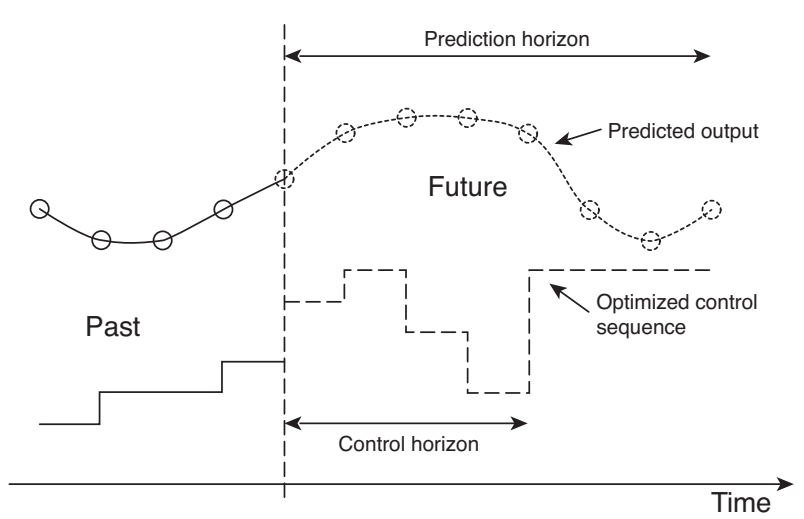

Figure 2

MPC strategy.

(NMPC) is the logic extension of MPC in which the linear model is substituted by a nonlinear one. Unfortunately, inserting this nonlinear model into the problem (1) leads in general to a non convex problem which may be difficult to solve, e.g. by a sequential approximation with Quadratic Programs at each time step, which is computationally expensive. Although efficient solvers for nonlinear programs exist (e.g. Bock et al., 2000), in this work we propose to apply a special nonlinear model class - the class of Linear Parameter Varying (LPV) systems - which allows to use an active set based QP solver in order to keep the computational burden low. Hence, the nonlinear system of the Diesel engine air path can be captured by a single model without a significant change in complexity for the online numerical solver.

\subsection{Determination of Multi-Linear Control Models}

As mentioned above, due to the nonlinear behavior in the wide operating range of a Diesel engine (e.g. engine speed from $800 \mathrm{rpm}$ to $4500 \mathrm{rpm}$ ) it is not possible to obtain a single linear model with a high precision. One evident possibility consists in working out several linear models to cover the whole operating range. A very new approach to accomplish this task is to use a clustering technique for the identification of piecewise affine systems (Ferrari-Trecate et al., 2003) or a more usual way is to divide the operating range empirically in small operating areas and identify several linear models (see Fig. 3).

The air path dynamics depend on many factors, but for the considered case of a warm engine it proved sufficient to consider besides the two manipulated variables (EGR and VGT) engine speed and injected fuel amount as model inputs, which shall be treated as measured disturbances. Neglecting the two measured disturbances would lead to unacceptable model quality. 


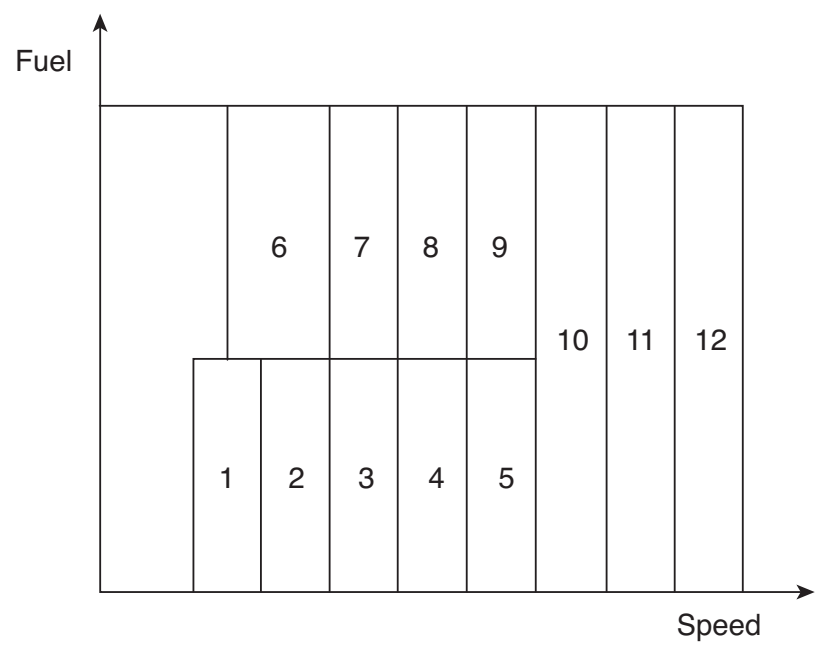

Figure 3

Segmentation of the engine working range.

As it will be shown in Section 2.3, for the determination of the linear control models a separate identification of state space models was done for the 12 regions, using a Prediction Error Method (PEM) (see e.g. Ljung, 1999).

\subsection{LPV Control Models}

In Wei (2006) a quasi-LPV model from the simplification of the physical model of a production Diesel engine was considered. The LPV model is defined by:

$$
A(z, \rho) y(k)=B(z, \rho) u(k)+m(k)
$$

Note that $A(z, \rho)$ and $B(z, \rho)$ represent polynomials in the shift operator $z$ and in the scheduling vector $\rho$, in contrast to equation (1) where $A$ and $B$ represent matrices. $m(k)$ is the error in the actual sampling instant. In a standard LPV case, $\rho$ is assumed to be an external variable, but in the quasi-LPV framework it may be a function of an internal state. The LPV system class has two strong advantages: it represents a continuous gain scheduling model and thus can approximate well many systems, and due to its structure and description of the input-output behavior, the parameters can be estimated from measured data by linear algorithms extended from the classical identification algorithms (Wei, 2006). Indeed, using:

$$
\begin{aligned}
\varphi_{k} & =\left[\begin{array}{llllllll}
-y_{k-1} & -y_{k-2} & \ldots & -y_{k-n} & u_{k-1} & u_{k-2} & \ldots & u_{k-m}
\end{array}\right] \\
\theta_{i} & =\left[\begin{array}{llllllll}
a_{i 1} & a_{i 2} & \ldots & a_{i n} & b_{i 1} & b_{i 2} & \ldots & b_{i m}
\end{array}\right]^{T} \\
F_{k} & =\left[\begin{array}{llllll}
1 & f_{1}\left(\rho_{1}, \rho_{2}, \ldots\right) & \ldots & f_{N-1}\left(\rho_{1}, \rho_{2}, \ldots\right)
\end{array}\right]
\end{aligned}
$$

where the functions $f_{1}\left(\rho_{1}, \rho_{2}, \ldots\right) \ldots f_{N-1}\left(\rho_{1}, \rho_{2}, \ldots\right)$ are design parameters, we can rewrite (2) as:

$$
\begin{aligned}
& y(k)=\left(F_{k} \otimes \varphi_{k}\right) \Theta+m_{k}=\Gamma_{k} \Theta+m_{k} \\
& \Theta=\left[\begin{array}{llll}
\left(\theta_{1}\right)^{T} & \left(\theta_{2}\right)^{T} & \ldots & \left(\theta_{N}\right)^{T}
\end{array}\right] \\
& \Gamma_{k}=\left(\begin{array}{ll}
F_{k} \otimes \varphi_{k}
\end{array}\right)
\end{aligned}
$$

(4) can be addressed by standard identification algorithms to estimate the parameter vector $\hat{\Theta}$ :

$$
\begin{aligned}
& \hat{\Theta}=\left(\Gamma^{T} \Gamma\right)^{-1} \Gamma^{T} Y \\
& \Gamma=\left[\Gamma_{1}, \Gamma_{2}, \ldots, \Gamma_{N_{m}}\right] \\
& Y=\left[y_{1}, y_{2}, \ldots, y_{N_{m}}\right]
\end{aligned}
$$

where $N_{m}$ is the number of measurements.

\subsection{MPC Formulation}

The formulation of MPC as Quadratic Program has already been shown in many works, for instance in Ferreau et al. (2006). In this work linear as well as LPV systems are considered, whereas linear systems can be considered as a special case of LPV. Hence, in the following the MPC algorithm formulation is presented for the LPV systems and, obviously, the same holds for the linear case with the only difference being constant system parameters. The parameters of the LPV system may change every sample, thus the recursive evaluation of the prediction model is: see Equation (6) This can be simplified to:

$$
X=\left(\begin{array}{ll}
S_{0} & S_{1}
\end{array}\right)\left(\begin{array}{l}
x_{0} \\
U
\end{array}\right)+V=S_{0} x_{0}+S_{1} U+V
$$

where $V$ is the disturbance matrix:

$$
V=\left(\begin{array}{c}
0 \\
Z v \\
\vdots \\
A_{N P H-1} \ldots A_{1} Z v+\ldots+Z v
\end{array}\right)
$$

and:

$$
S_{0}=\left(\begin{array}{c}
I \\
A_{0} \\
\vdots \\
A_{N P H-1} \cdots A_{0}
\end{array}\right)
$$

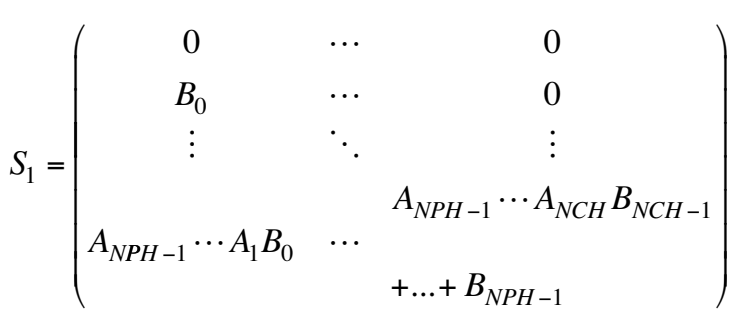




$$
\begin{aligned}
\left(\begin{array}{c}
x_{0} \\
x_{1} \\
\vdots \\
x_{N P H}
\end{array}\right) & \left(\begin{array}{cccc}
I & 0 & \cdots & 0 \\
A_{0} & B_{0} & \cdots & 0 \\
\vdots & \vdots & \ddots & \vdots \\
A_{N P H-1} \cdots A_{0} & A_{N P H-1} \cdots A_{1} B_{0} & \cdots & A_{N P H-1} \cdots A_{N C H} B_{N C H-1} \\
& \\
0 & \\
Z v & \\
\vdots \\
A_{N P H-1} \cdots A_{1} Z v+\ldots+Z v
\end{array}\right) \\
& +\left(\begin{array}{c}
u_{N C H-1}
\end{array}\right)
\end{aligned}
$$

Substituting (7) into (1) leads to:

$$
\begin{gathered}
\min _{U} \frac{1}{2} U_{k}^{T}\left(S_{1}^{T} Q_{\text {diag }} S_{1}+R_{\text {diag }}\right) U_{k}+U_{k}^{T} S_{1}^{T}\left(S_{0} x_{0}+V-X_{\text {ref }}\right) \\
X_{\text {ref }}=I \cdot x_{\text {ref }} \\
Q_{\text {diag }}=\operatorname{diag}(Q, Q \cdots Q) \\
R_{\text {diag }}=\operatorname{diag}(R, R \cdots R) \\
U=\left(u_{1} ; \cdots, u_{N C H}\right) \\
X=\left(x_{0} ; x_{1} ; \cdots, x_{N P H}\right)
\end{gathered}
$$

which can be solved using standard QP solvers. As for the LPV case the matrices $A$ and $B$ depend on the scheduling vector, whereas they are updated according to the result of the previous optimization step and the problem (10) is set up again each sampling instant. Note again, that in the special case of linear systems the matrices $A$ and $B$ are constant, which results in a single QP for all times and makes the QP reformulation at every time instant unnecessary.

\subsection{State Estimation}

For the application of state space MPC, information about the system state is necessary to obtain the optimal control sequence for the next time instant. For the presented results, a Kalman filter (Kalman, 1960) was used to estimate the current system states based on a system model and measured in- and output signals. In the following, the basic ideas are briefly summarized.

Considering a linear discrete time system description:

$$
\begin{gathered}
x_{k+1}=A \cdot x_{k}+B \cdot u_{k}+G \cdot w_{k} \\
y_{k}=C \cdot x_{k}+D \cdot u_{k}+H \cdot w_{k}+v_{k}
\end{gathered}
$$

with the input $u$, the output $y$, a process noise input $w$ and a measurement noise $v$, it is assumed that both noise inputs are white and with normal probability distribution $\left(E\left\{w_{k}\right\}=\right.$ $\left.E\left\{v_{k}\right\}=0\right)$. Although in practice the noise covariances:

$$
E\left\{w_{k} \cdot w_{k}^{T}\right\}=Q \quad E\left\{v_{k} \cdot v_{k}^{T}\right\}=R \quad E\left\{w_{k} \cdot v_{k}^{T}\right\}=N
$$

might change, for the following it is assumed that they are constant. Then, the Kalman filter constructs a state estimate $\hat{x}_{k}$ that minimizes the steady state error covariance:

$$
P=\lim _{k \rightarrow \infty} E\left\{\left(x_{k}-\hat{x}_{k}\right) \cdot\left(x_{k}-\hat{x}_{k}\right)^{T}\right\}
$$

The implementation of the algorithm can be split into two parts, a prediction and an update phase. During the first phase the filter predicts the future system state, based on the current state and the system input signals. The expected error covariance matrix $P$ is calculated using this information:

$$
\begin{aligned}
& \hat{x}_{k+1 \mid k}=A \cdot \hat{x}_{k \mid k}+B \cdot u_{k} \\
& P_{k+1 \mid k}=A \cdot P_{k, k} A^{T}+Q
\end{aligned}
$$

During the update phase the error between predicted system outputs and measured, but noisy, signals is used to correct the filter gain and update the current state prediction:

$$
\begin{aligned}
\bar{H}_{k} & =P_{k \mid k-1} \cdot C^{T}\left[C \cdot P_{k \mid k-1} \cdot C^{T}+R\right]^{-1} \\
\hat{x}_{k, k} & =\hat{x}_{k \mid k-1}+\bar{H}_{k} \cdot\left(y_{k}-\hat{y}_{k}\right) \\
P_{k, k} & =\left[I-\bar{H}_{k} \cdot C\right] \cdot P_{k \mid k-1}
\end{aligned}
$$

Since in most cases the error covariances are not known exactly, the matrices $Q$ and $R$ can be seen as tuning factors, which either can be found with adaptive methods, or need to be determined by empirical tests. For the considered nonlinear models of the NMPC framework an Extended Kalman 


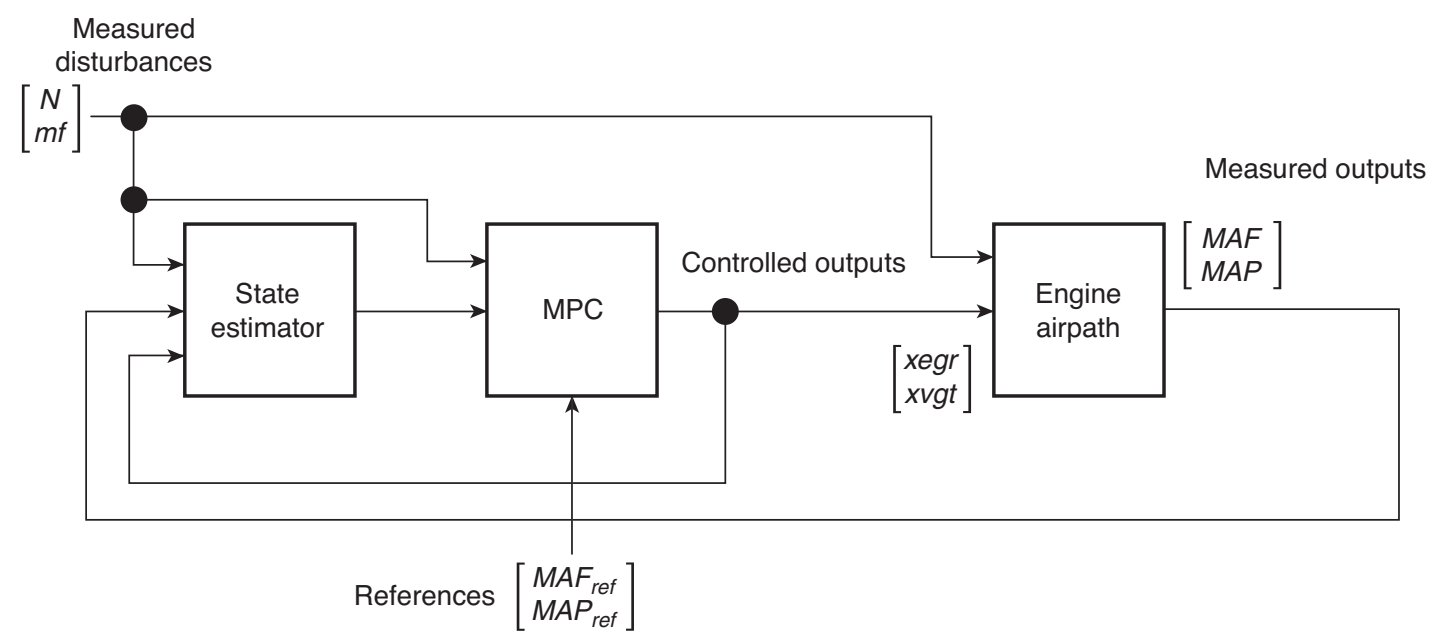

Figure 4

Closed-loop control structure.

Filter (EKF) was used for state estimation. Based on a generic nonlinear system of the given structure:

$$
\begin{aligned}
& x_{k+1}=f\left(x_{k}, u_{k}, k\right)+w_{k} \\
& y_{k}=g\left(x_{k}, u_{k}, k\right)+v_{k}
\end{aligned}
$$

and with the same assumptions on the noise characteristics as for the linear plant model, the Kalman filter update equations remain identical as above, only the system matrices $A$ and $C$ are now derived by a linearization in the Operating Point $(O P)$ of the system at each time instant:

$$
\begin{aligned}
& A\left(k, x_{k}, u_{k}\right)=\left.\frac{\partial f\left(x_{k}, u_{k}, k\right)}{\partial x_{k}}\right|_{O P} \\
& C\left(k, x_{k}, u_{k}\right)=\left.\frac{\partial g\left(x_{k}, u_{k}, k\right)}{\partial x_{k}}\right|_{O P}
\end{aligned}
$$

To take into account steady state offsets the control model is augmented with an output disturbance model (Muske and Badgwell, 2002). In the linear case the augmented system can be described by:

$$
\begin{aligned}
& {\left[\begin{array}{l}
x_{k+1} \\
e_{k+1}
\end{array}\right]=\left[\begin{array}{cc}
A_{\text {plant }} & 0 \\
0 & I
\end{array}\right] \cdot\left[\begin{array}{l}
x_{k} \\
e_{k}
\end{array}\right]+\left[\begin{array}{c}
B_{\text {plant }} \\
0
\end{array}\right] \cdot u_{k}} \\
& y_{k}=\left[\begin{array}{ll}
C_{\text {plant }} & I
\end{array}\right] \cdot\left[\begin{array}{l}
x_{k} \\
e_{k}
\end{array}\right]+D_{\text {plant }} \cdot u_{k}
\end{aligned}
$$

where for each measured output the disturbance model is extended with an additional state.

\subsection{Control Structure}

Both, the multi-linear MPC and the LPV based NMPC closed loop systems can be described by the control structure presented in Figure 4. The MPC is provided with the estimated states, the measured disturbance and of course the reference set points. The estimated states are obtained by a standard Kalman filter in the linear case or an EKF in the nonlinear application.

\section{MODEL PREDICTIVE ENGINE CONTROL}

\subsection{Diesel Engine Air Path}

The air path of a modern Diesel engine is a complex thermodynamic and mechanic system. The schematic diagram of a turbocharged Diesel engine with high pressure Exhaust Gas Recirculation $(E G R)$ is illustrated in Figure 5. The inlet and exhaust manifolds of a Diesel engine are coupled twice firstly through the Variable Geometry Turbocharger (VGT) and secondly through the Exhaust Gas Recirculation (EGR) path.

In this system key influent factors on $M A F$ and $M A P$ are positions of EGR and $V G T$, which are the considered control input variables in this work. Since both actors affect both output variables, engine air path control is indeed a MIMO control problem. The actual standard is to apply a heuristically tuned feedforward part in combination with two SISO (PID) feedback controllers. Typically, most of the control action is generated by the with high effort determined feedforward, while the task of the feedback controllers is mainly to 


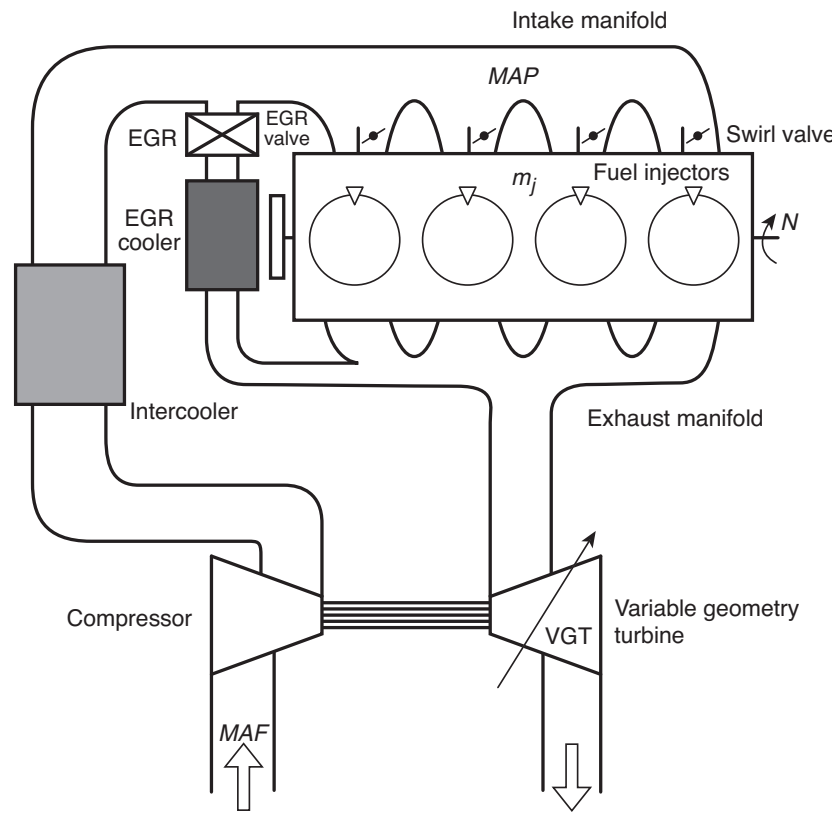

Figure 5

Structure of a Diesel engine air path.

compensate production tolerances, aging and wear as well as to reject disturbances. In the following, as an alternative to the standard method the application of the MPC approaches is presented, using EGR valve position (constrained from 0 to $100 \%$ ), VGT position (constrained from 0 to $100 \%$ ) as control inputs and engine speed $N$ and injected fuel per stroke $m_{f}$ as measured disturbance $v$. The control task is to track references for the two control outputs $M A F$ in $\mathrm{kg} / \mathrm{h}$ and $M A P$ in $\mathrm{hPa}$.

\subsection{Real Time System Setup}

Figure 6 depicts the real time implementation setup of the MPC approaches. The control design was done in Matlab/ Simulink and finally the real time controller was implemented on a rapid prototyping system. In order to get control authority of the air path, a development Engine Control Unit was used, which allowed to bypass the standard air path control loops while all the remaining engine control loops were kept at their standard.

\subsection{Implementation of Multi-Linear MPC}

According to the segmentation of Figure 3 several linear models were determined independently. For identification the choice of excitation signals and patterns for MIMO identification is known to be critical (Gevers et al., 2006). Figure 7 presents the applied identification input signals as well as the measured system outputs. Second order PEM models turned out to be sufficient for the restricted operating ranges, e.g. Figure 8 shows the satisfactory validation results of the model for region 5 .

The engine speed and the injected fuel amount were considered as measured disturbances $v$ for the control model and furthermore an output error disturbance model $e$ was applied in order to guarantee offset free tracking, leading to:

$$
\begin{aligned}
& {\left[\begin{array}{l}
x_{k+1} \\
u_{k+1} \\
e_{k+1}
\end{array}\right]=\left[\begin{array}{ccc}
A_{M A F, M A P} & B_{M A F, M A P} & 0 \\
0 & I & 0 \\
0 & 0 & I
\end{array}\right] \cdot\left[\begin{array}{l}
x_{k} \\
u_{k} \\
e_{k}
\end{array}\right]+\left[\begin{array}{ll}
I & 0 \\
0 & I \\
0 & 0
\end{array}\right] \cdot\left[\begin{array}{c}
v_{k} \\
\Delta u_{k}
\end{array}\right]} \\
& y_{k}=\left[\begin{array}{lll}
C_{M A F, M A P} & 0 & I
\end{array}\right] \cdot\left[\begin{array}{c}
x_{k} \\
u_{k} \\
e_{k}
\end{array}\right]
\end{aligned}
$$

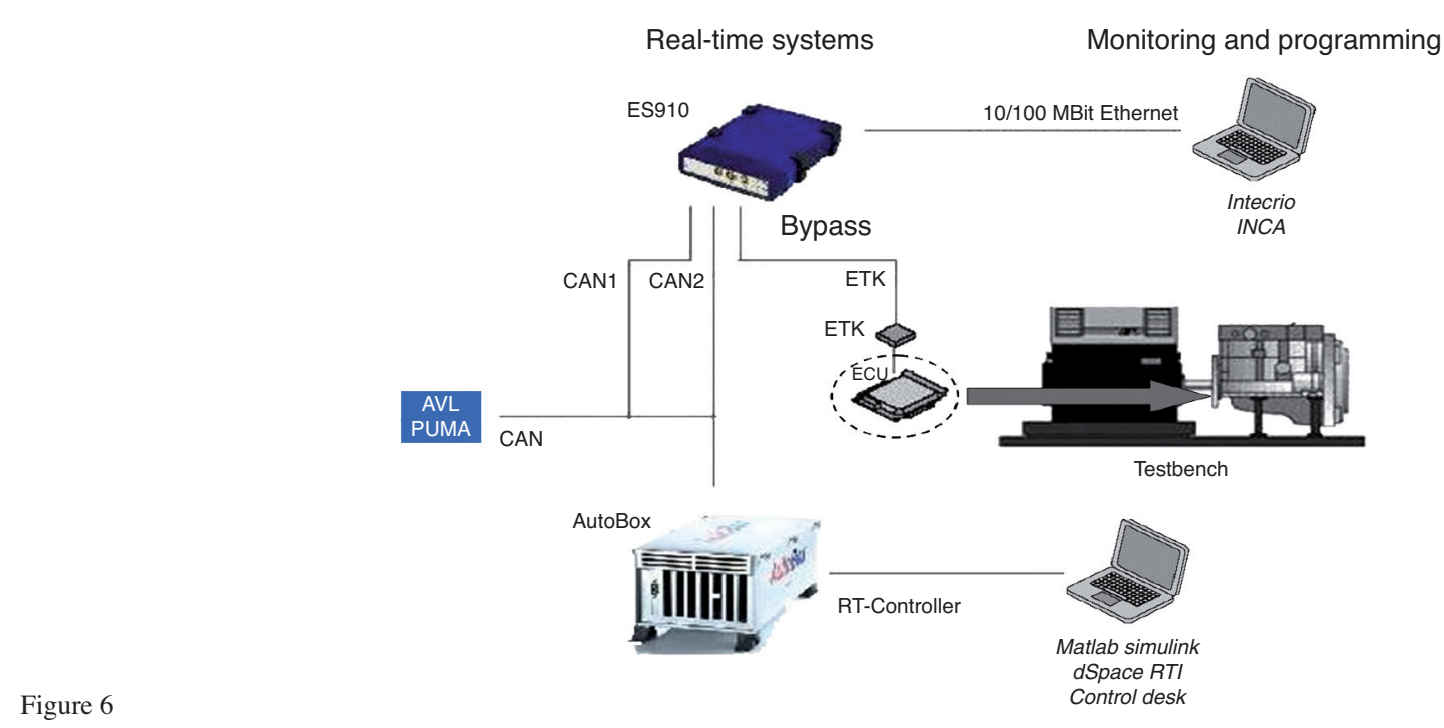

Schematic representation of the combustion engine test bench. 

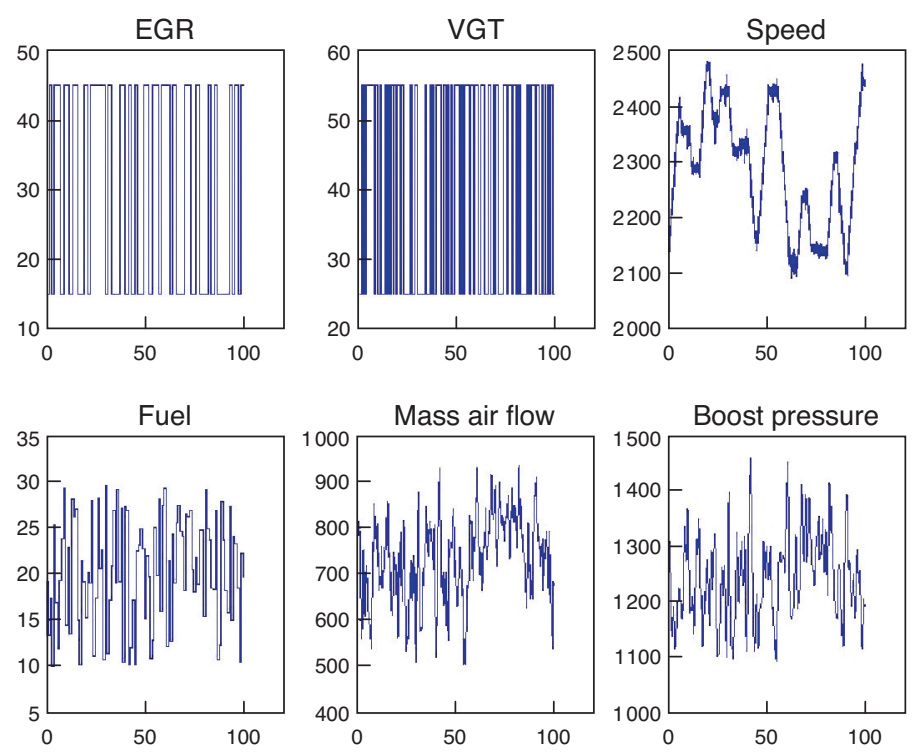

Figure 7

Identification signals (\%, \%, rpm, mg/stroke, mg/stroke, hPa $v s$ time).
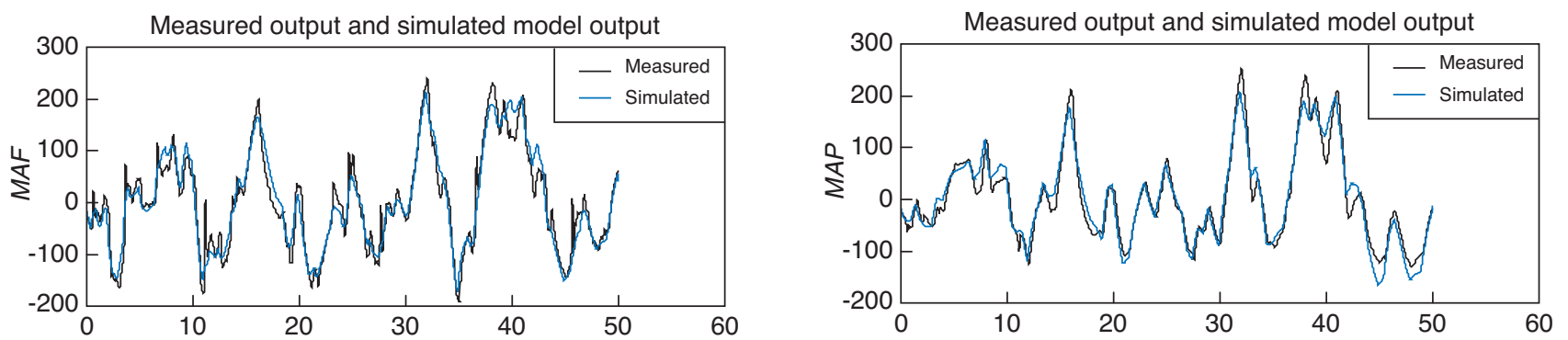

Figure 8

Validation of model 5 (mg/stroke and hPa $v s$ time, mean value compensated).

where:

$$
x_{k} \in R^{2}, u_{k} \in R^{2}, e_{k} \in R^{2}, \Delta u_{k} \in R^{2} \text { and } v_{k} \in R^{2}
$$

Based on the identified models, in the next step, Kalman filters as well as linear Model Predictive Controllers were designed for each region. For the actual case explicit MPC was selected, where affine control laws are computed a priori for every possible initial state and consequently no QP solver is required in the real-time implementation (see Bemporad et al. (2002) for more details on explicit MPC). Note that the final control signal of this explicit version is the same as an online optimizer would obtain ${ }^{2}$ (see Tab. 1 for the applied parameter settings). Finally the multiple controllers according to Figure $4^{3}$ have been implemented on a real time system (Fig. 6),

\footnotetext{
${ }^{2}$ Subject to applying the same linear system as well as identical constraints and an equal tuning.

${ }^{3}$ The block "MPC" of Figure 4 contains the affine control laws.
}

TABLE 1

MPC tuning parameters

\begin{tabular}{c|c}
\hline Property & Value \\
\hline$Q_{M P C}$ & {$[0.50 ; 00.5]$} \\
\hline$R_{M P C}$ & {$\left[\begin{array}{lll}1 & 0 ; 0 & 1\end{array}\right]$} \\
\hline$\Delta u_{M P C}$ & {$[-10-5 ; 3.35]$} \\
\hline$N P H$ & 120 \\
\hline$N C H$ & 1 \\
\hline$T_{s}$ & $0.05 \mathrm{~s}$ \\
\hline
\end{tabular}

whereas a controller output switching was realized. Therefore, only a single controller - the one belonging to the actually effective segment according to Figure 3 - was in closed loop control authority. In order to provide a smooth transition between the segments, a continuous update of all 
state estimators with the current system input was done at every time instant.

\subsection{Implementation of LPV-NMPC}

As presented in Section 1.2, the proposed LPV identification algorithm has been used to model the air path system. It turned out that using $V G T$ and $N$ as scheduling parameters $\rho_{1}$ and $\rho_{2}$ is a valuable choice. The MAF model and the MAP model each consist of four parallel transfer functions (one for each input) of the type:

$$
G_{i}(z, \rho)=\frac{b_{i}\left(\rho_{1}, \rho_{2}\right)}{z+a_{i}\left(\rho_{1}, \rho_{2}\right)}
$$

with the following coefficients:

$$
\begin{gathered}
a_{i}\left(\rho_{1}, \rho_{2}\right)=a_{i}(V G T, N)=a_{i 1}+a_{i 2} \cdot V G T+a_{i 3} \cdot N+a_{i 4} \cdot V G T \cdot N \\
b_{i}\left(\rho_{1}, \rho_{2}\right)=b_{i}(V G T, N)=b_{i 1}+b_{i 2} \cdot V G T+b_{i 3} \cdot N+b_{i 4} \cdot V G T \cdot N \\
\forall i \in[1,2,3,4]
\end{gathered}
$$

In Figure 9 as an example the described structure is depicted for the MAP part of the LPV model. The model inputs are the

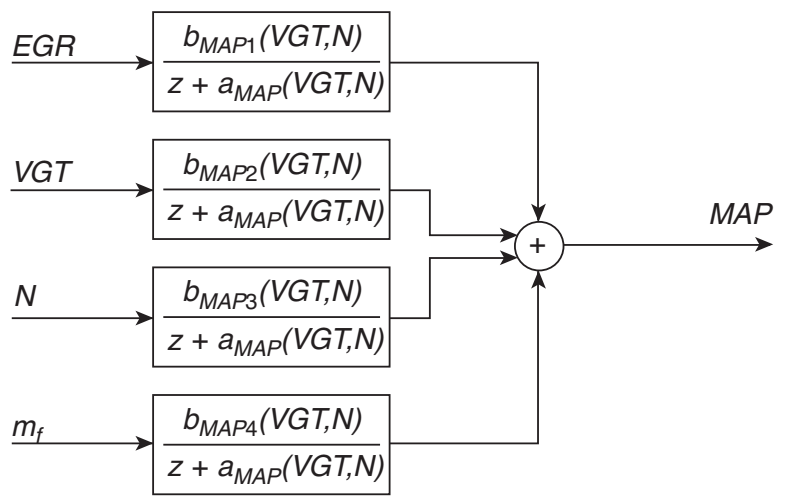

Figure 9

Structure of the LPV model for MAP.

engine speed $N$, the injected fuel amount $m_{f}$ and the position of the EGR and VGT actuators.

The sampling time of the identification data was $T_{S}=0.05 \mathrm{~s}$. As already mentioned the model is a combination of two MISO systems (one for $M A F$ and one for $M A P$ ) which were identified separately. For the excitation of the inputs $E G R$ and $V G T$ and the external disturbances $N$ and $m_{f}$ white noise signals with different sample times were used around a specified mean value which is shown in Figure 10. To avoid a
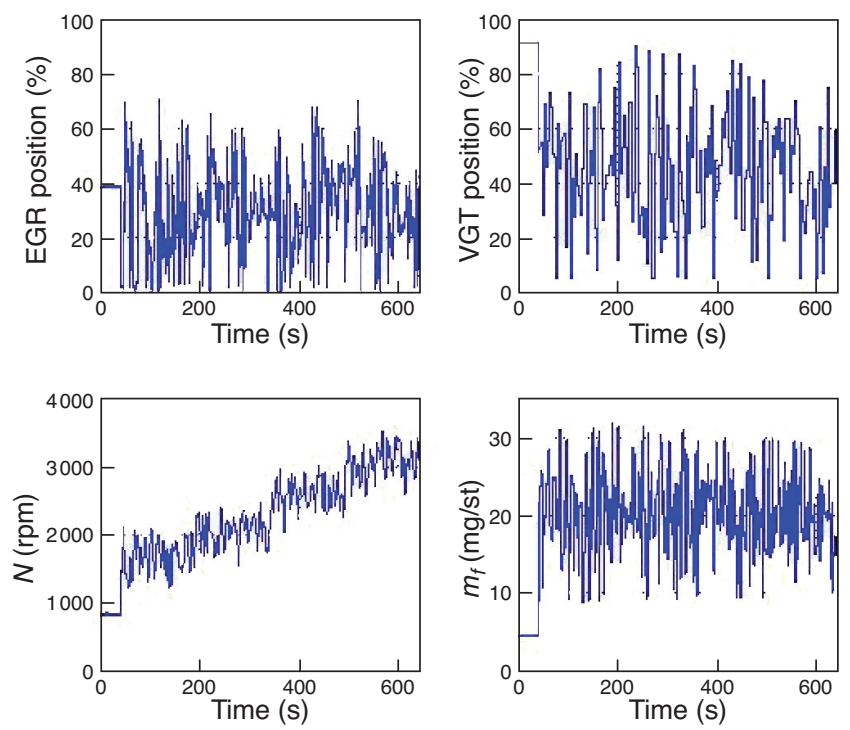

Figure 10

Used input excitation signals for the LPV air path model identification.

damage of the engine, caused by too high manifold air pressures, the maximum $V G T$ position was constrained at higher engine speeds.

Figure 11 shows the satisfactory validation results of the identified model for the two outputs $M A P$ and $M A F$.

TABLE 2

LPV model quality

\begin{tabular}{c|c}
\hline & $V A F$ \\
\hline$M A F$ & $89.46 \%$ \\
\hline$M A P$ & $77.88 \%$ \\
\hline
\end{tabular}

In Table 2 the $V A F$ (Variance Accounted For) is used as model quality criterion. The $V A F$ is defined as:

$$
V A F=\max \left(1-\frac{\operatorname{var}\left(y_{k}-\hat{y}_{k}\right)}{\operatorname{var}\left(y_{k}\right)}\right) \cdot 100[\%]
$$

Transforming the two MISO models from the transfer function model into state-space form yields to description (19) with non constant matrices $A_{M A F, M A P}, B_{M A F, M A P}, C_{M A F, M A P}$, where:

$x_{k} \in R^{8}, u_{k} \in R^{2}, e_{k} \in R^{2}, \Delta u_{k} \in R^{2} \quad$ and $\quad v_{k} \in R^{2}$

The implementation of the closed-loop control structure in Figure 4 has been done using the setup shown schematically in Figure 6, whereas the NMPC was designed in $\mathrm{C}++$ and 

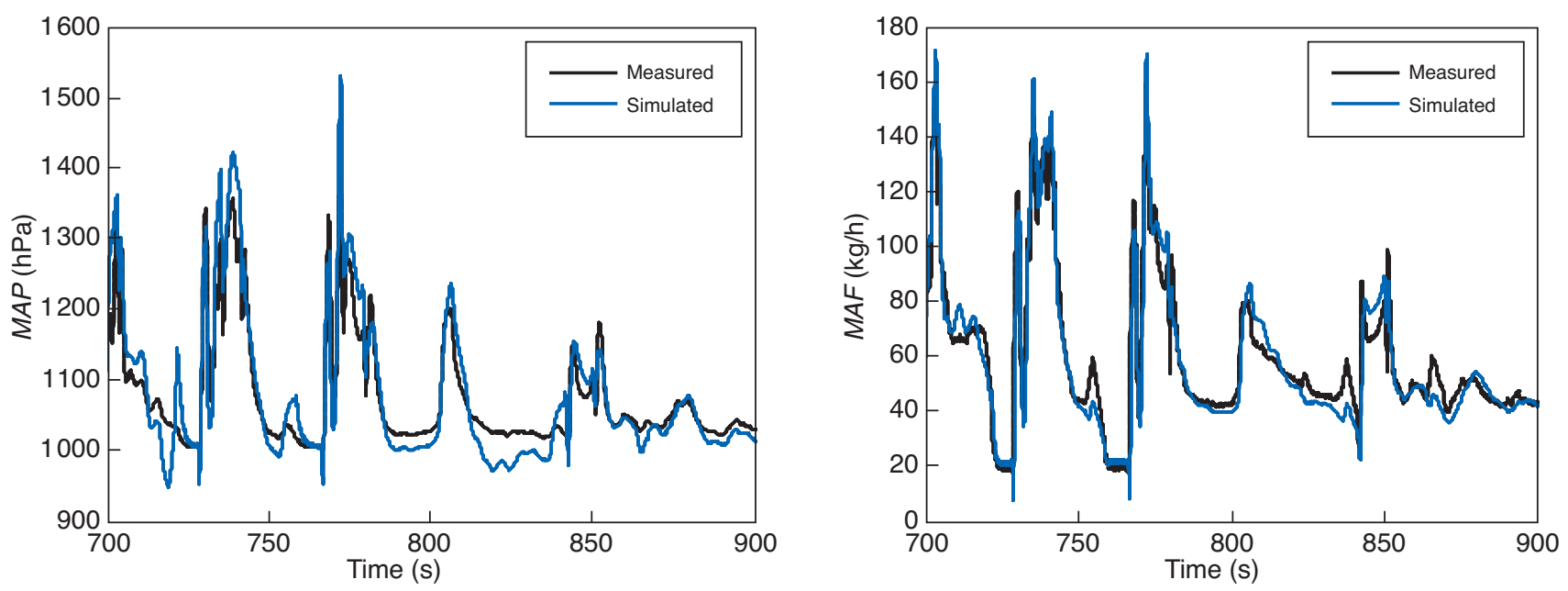

Figure 11

Validation result for $M A P$ and $M A F$.

implemented on the rapid prototyping hardware system. For solving the resulting QP online, the software package qpOASES was applied (Ferreau, et al., 2008), whereas in the general nonlinear case a nonlinear program (NLP) instead of a QP has to be treated. However as already mentioned above, for the considered case of the LPV model formulation the problem can be stated as a QP at every time instant, which again can be treated by a standard QP solver. Further information about different solution variants for special QPs can be found in Ferreau et al. $(2006,2008)$. The inputs (EGR, $\left.V G T, N, m_{f}\right)$ and outputs $(M A F, M A P)$ of the engine were measured together with the model states by the EKF. The initial setup of tuning parameters is shown in Table 3.

TABLE 3

NMPC tuning parameters

\begin{tabular}{c|c}
\hline Property & Value \\
\hline$Q_{N M P C}$ & {$\left[\begin{array}{lll}10 ; 0 & 1\end{array}\right]$} \\
\hline$R_{N M P C}$ & {$[200 ; 020]$} \\
\hline$\Delta u_{N M P C}$ & {$[-5-5 ; 55]$} \\
\hline$N P H$ & {$[60,80,100,120,140,160]$} \\
\hline$N C H$ & 3 \\
\hline$R_{\mathrm{EKF}}$ & {$[10 ; 01]$} \\
\hline$Q_{\mathrm{EKF}}$ & $*$ \\
\hline$T_{s}$ & $0.05 \mathrm{~s}$ \\
\hline
\end{tabular}

* The last two terms of $Q_{\mathrm{EKF}}$ (errors of $M A F$ and $\left.M A P\right) Q_{\mathrm{EKF}}(9,9)$ and $Q_{\mathrm{EKF}}(10,10)$
are equal to $10^{6}$, the other terms on diagonal are $10^{-2}$, for the purpose of offset free tracking.
Note that, due to the nonlinearity, it is difficult to choose the exact horizons that ensure feasibility and stability of the optimization problem. In order to enforce the closed-loop stability, a conservative choice is to set the prediction horizon much longer than the control horizon (see e.g. Chen and Allgöwer, 1998).

\section{EXPERIMENTAL RESULTS}

\subsection{Multi-linear MPC Results}

Figure 12a depicts a comparison of the multi-linear MPC against a standard control setup during reference changes of $M A F$. As it can be seen, in particular during the transients the MPC shows a much faster reaction and consequently achieves a significant improvement of the dynamical response. In Figure $12 \mathrm{~b}$ steps of engine speed during constant $M A P / M A F$ references were applied. The multi-linear MPC was able to satisfactorily suppress the output impact of the disturbance steps, also during region transitions.

\subsection{NMPC Results}

\subsubsection{Choice of Control and Prediction Horizon}

The computational burden is an important issue for the real time implementation. In particular the choice of the control horizon has a high impact on the required computation time, whereas the feasible maximum was limited to $\mathrm{NCH}=3$. To 

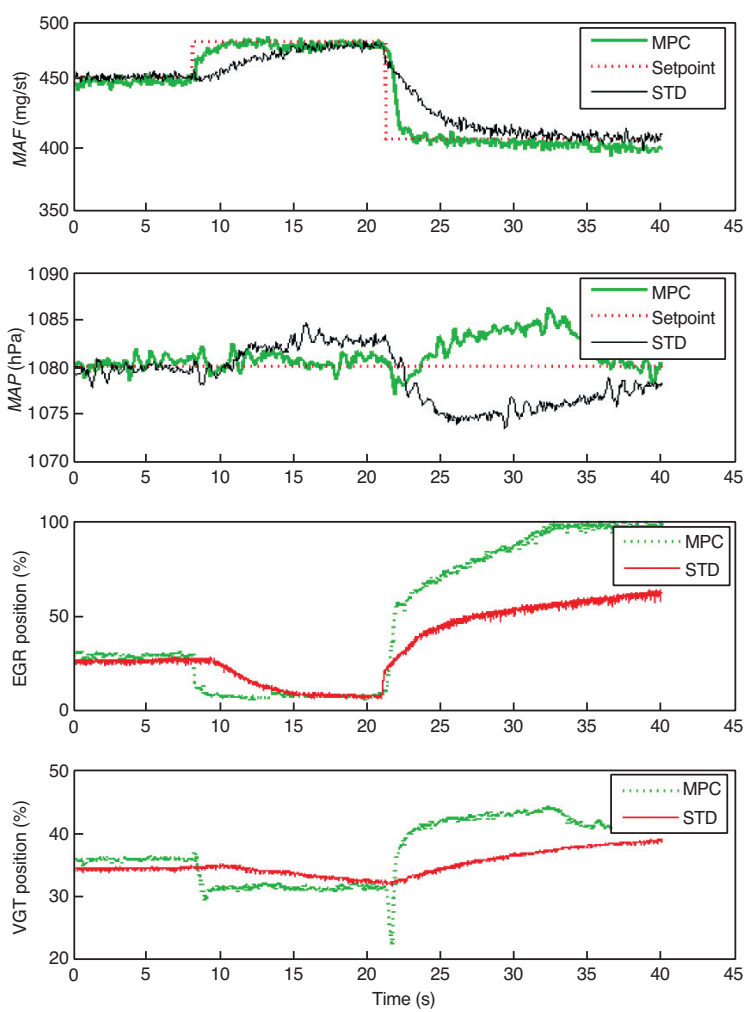

a)
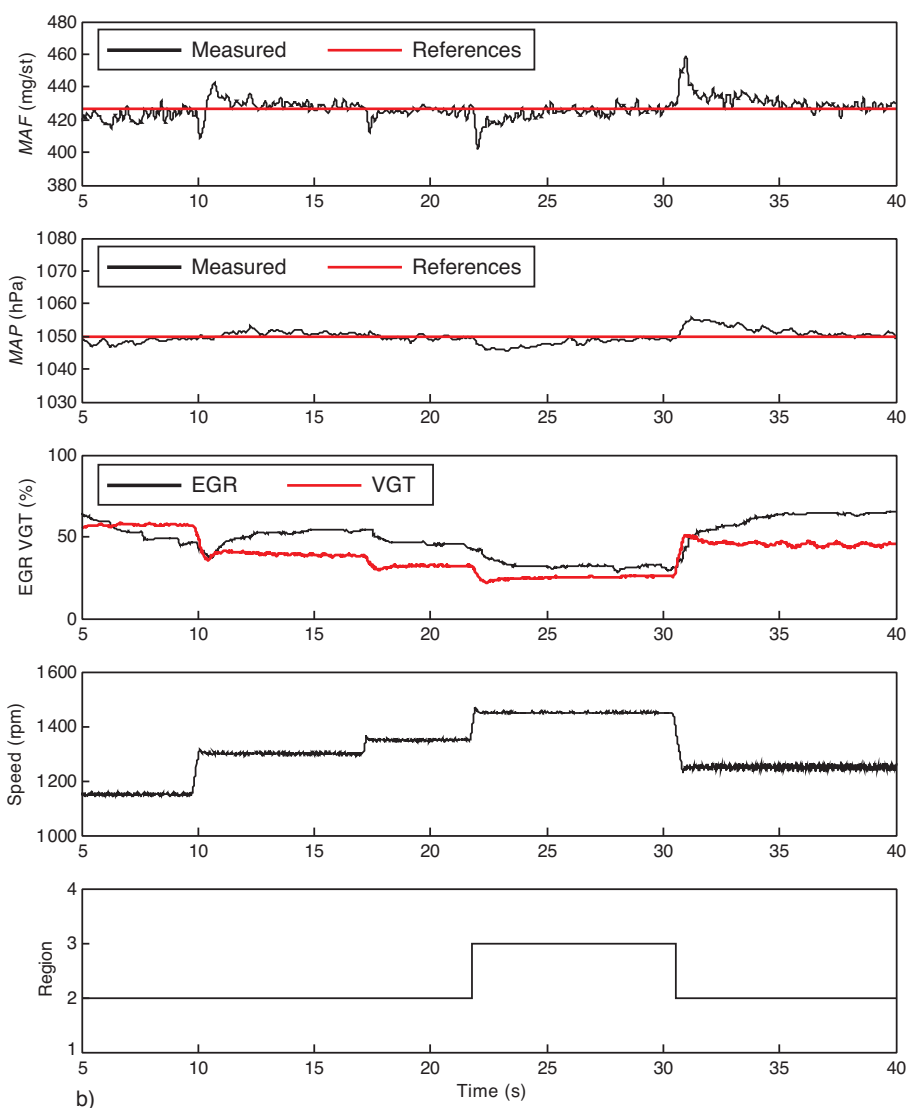

Figure 12

Multi-linear MPC evaluation during reference changes a) and region transitions b).

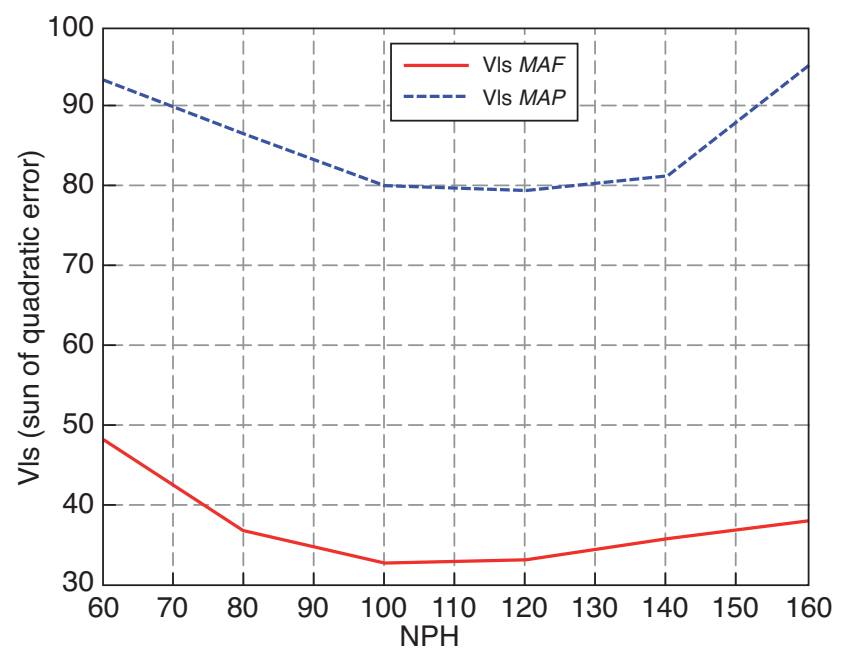

Figure 13

Relative sum of squared errors $V_{I s}$ for the tracking of $M A P$ and $M A F$ as a function of the prediction horizon $(\mathrm{NCH}=3)$. analyze the significance of the prediction horizon, several MPCs with equal $\mathrm{NCH}$ and different $\mathrm{NPH}$ were tested (see $T a b .3$ ). The comparison was done on a test sequence and the tracking errors for $M A F$ and $M A P$ were used for evaluation (relative sum of squared errors). Figure 13 depicts the change in performance with different prediction horizons, whereas $N P H=120$ gave the best results for the considered case ${ }^{4}$.

\subsubsection{Performance Comparison of NMPC to the Standard Control Setup}

To show the feasibility of the Nonlinear MPC approach, it was compared against the standard control setup during reference changes of $M A F$ and MAP. Figure 14 depicts the results and Table 4 presents a comparison of the cumulative tracking error during the changes. Although a difference in the control signals can be noticed, the overall tracking performance is similar - while NMPC had advantages in MAF tracking, the

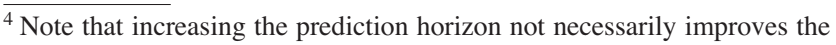
control performance of MPC.
} 

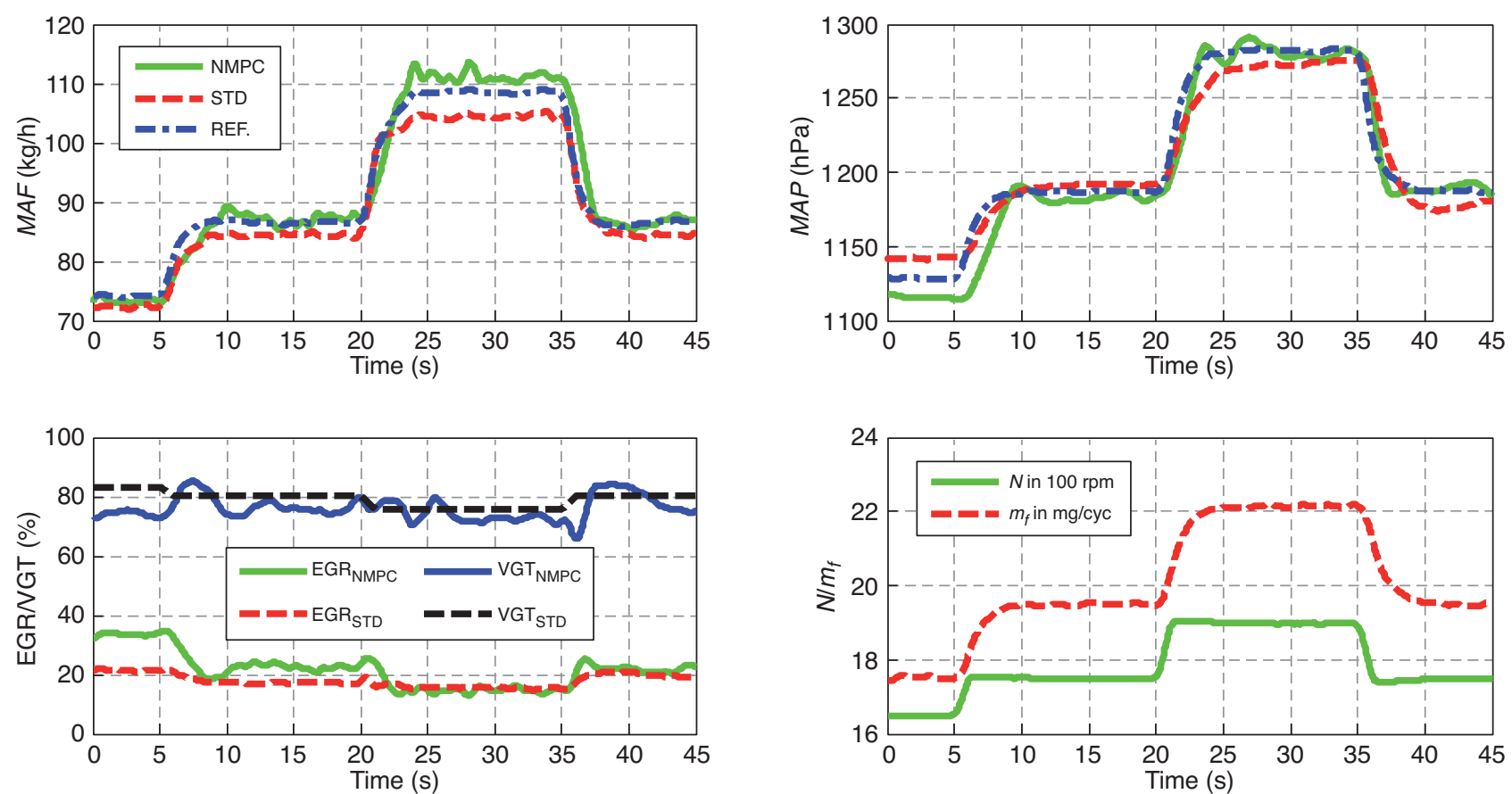

Figure 14

Comparison of NMPC with the standard setup for a reference change.

standard control had a slightly better MAP tracking result. Here two issues have to be mentioned:

- The focus of the LPV-NMPC approach was on providing a basic and straight forward method. Therefore, by intention performance was sacrificed in order to keep the complexity low (e.g. by identifying very simple LPV models).

- The comparison of both controllers was done in a region where the standard calibration already performed well. Obviously any controller cannot rule out the physics - if the standard setup is already close to the optimum, even the best NMPC is not able to show significant performance gains.

\section{TABLE 4}

Tracking performance comparison (sum of relative quadratic error)

\begin{tabular}{c|c|c}
\hline & NMPC & STD \\
\hline$e_{M A F}$ & $77 \%$ & $100 \%$ \\
\hline$e_{M A P}$ & $107 \%$ & $100 \%$ \\
\hline
\end{tabular}

\subsubsection{Performance Comparison of NMPC to MPC}

In a next step the NMPC is compared to a single linear MPC to provide insight in the difference between both approaches and information whether the nonlinear extension leads to better results than a single linear MPC. To this end the same setup was used for a linear framework, i.e. equal weights and horizons in the formulation (1). Even the same identified model as for the NMPC was applied, however for the linear plant model the parameters (coefficients $a_{\mathrm{i}}$ and $b_{\mathrm{i}}$ of $E q .20$ ) were kept at constant values. Moreover, it should be noted that during this comparison only one linear MPC was used and not a multi-linear MPC as presented in Section 3.1.

TABLE 5

Tracking performance comparison (sum of relative quadratic error)

\begin{tabular}{c|c|c}
\hline & NMPC & Linear MPC \\
\hline$e_{M A F}$ & $49 \%$ & $100 \%$ \\
\hline$e_{M A P}$ & $14 \%$ & $100 \%$ \\
\hline
\end{tabular}

In Table 5, the tracking performance for both target quantities is shown for the reference change depicted in Figure 15. Obviously, the single model of the linear MPC represents the nonlinear system well only in a restricted operating range. Therefore, the control performance of the NMPC is significantly better than the MPC during the considered transient. Here it should be kept in mind that even the steady state gains 

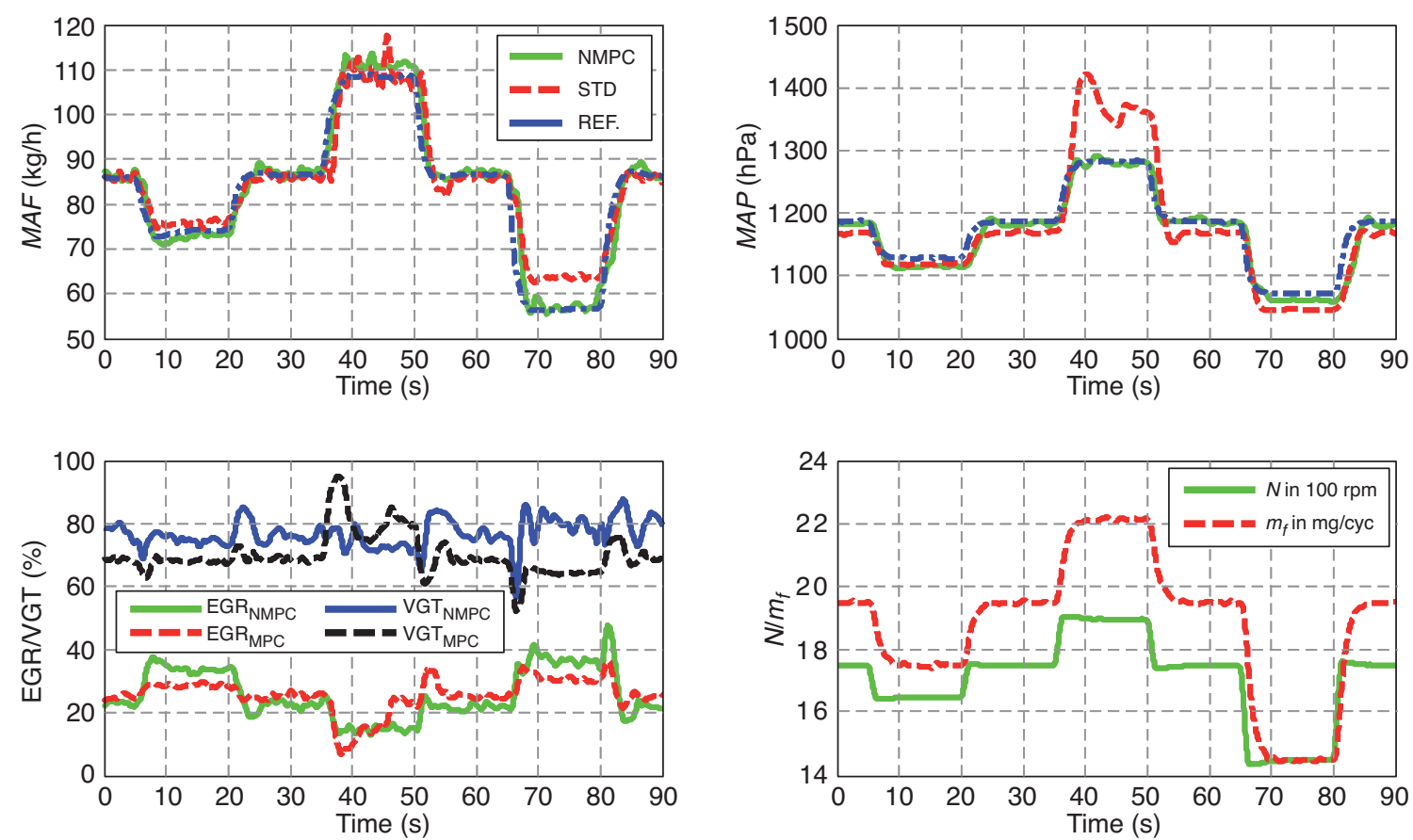

Figure 15

Comparison of NMPC with MPC for a reference change.

change over the operating range (see. Fig. 1) and if such a change occurs the linear MPC will use an incorrect model for prediction, thus leading to a poor performance (e.g. between $t=37 \mathrm{~s}$ and $t=53 \mathrm{~s}$ ).

\section{CONCLUSIONS}

Two different frameworks for Model Predictive Control of a Diesel engine air path have been presented, namely a multilinear MPC and a NMPC approach. The main idea was to present practically relevant methods providing a simple and straight forward setup and yielding a similar performance than a with a huge effort heuristically determined standard control setup.

Compared to the standard control in the evaluation scenario an improvement of the multi-linear MPC tracking performance was achieved especially during fast transients. A drawback of the multi linear MPC is that all controllers might have to be designed separately to reach the best performance and therefore increase the tuning effort.

For the NMPC strategy simple Linear Parameter Varying models proved sufficient to capture the essential nonlinearity of the system. The LPV identification as well as the NMPC were successfully applied to the engine and show a similar performance of the MAF/MAP tracking than the well working standard control. Although further improvements would be possible with the NMPC setup, in view of the lowest feasible complexity, performance was sacrificed to keep the tuning effort as low as possible. In this way, predictive control strategies (NMPC and multi linear MPC) might become attractive for a possible future use in production engine control.

The future work will focus on a further enhancement of the LPV identification scheme and a possible self tuning implementation of the NMPC. With the continuously increasing computational power of the available hardware, more complex model structures and also higher control horizons for the MPC can soon be tested, which lets us expect an increase in the possible performance compared to the current implementation. Another interesting and possible valuable extension of the proposed framework will be the combination with a moving horizon observer for state estimation.

\section{ACKNOWLEDGMENTS}

The authors gratefully thank the support from Richard Fuerhapter during the engine tests as well as Hans Joachim 
Ferreau for the QP solver issues. The sponsoring of this work by the COMET K2 Center "Austrian Center of Competence in Mechatronics (ACCM)" is gratefully acknowledged. The COMET Program is funded by the Austrian Federal Government, the Federal State Upper Austria and the Scientific Partners of ACCM.

\section{REFERENCES}

Ayadi M., Langlois N., Chafouk H. (2004) Polynomial control of nonlinear turbocharged diesel engine model, IEEE International Conference on Industrial Technology 1384-1389.

Bemporad A., Morari M., Dua V., Pistikopoulos E.N. (2002) The explicit linear quadratic regulator for constrained systems, Automatica 38, 3-20.

Bock H.G., Diehl M., Leineweber D.B., Schlöder J.P. (2000) A direct multiple shooting method for real-time optimization of nonlinear DAE processes, Prog. Systems Theory 26, 246-267.

Chen H., Allgöwer F. (1998) A computationally attractive nonlinear predictive control scheme with guaranteed stability for stable systems, J. Process Control 8, 475-485.

Ferrari-Trecate G., Musellic M., Liberati D., Morari M. (2003) A clustering technique for the identification of piecewise affine systems, Automatica 39, 205-217.

Ferreau H.J., Bock H.G., Diehl M. (2006) Real-Time Iterations and Online Active Set Strategies for Fast Linear and Nonlinear MPC, Nonlinear Model Predictive Control Workshop for fas Systems 2006, 13-22. IFAC, Grenoble, France.

Ferreau H.J., Ortner P., Langthaler P., del Re L., Diehl M. (2007) Predictive Control of a real-world Diesel engine using an extended online active set stragegy, Annu.Rev. Control 31, 293-301.

Ferreau H.J., Bock H.G., Diehl M. (2008) An online active set strategy to overcome the limitations of explicit MPC, Int. J. Robust Nonlinear Control 18, 816-830.

Garcia-Ortiz J.V. (2004) Aportación a la mejora del control de la gestión de aire en motores Diesel turboalimentados mediante distintos algortimos de control. Departamento de máquinas y motores térmicos, Universidad Politécnica de Valencia, Valencia.

Gevers M., Miskovíc L., Bonvin D., Karimi A. (2006) Identification of multi-input systems: variance analysis and input design issues, Automatica 42, 559-572.

Herceg M., Raff T., Findeisen R., Allgöwer F. (2006) Nonlinear Model Predictive Control of a Turbocharged Diesel Engine, International Conference on Control Applications, Munich.
Jankovic M., Jankovic M., Kolmanovsky I. (2000) Constructive Lyapunov Control Design for Turbocharged Diesel Engines, IEEE Trans. Control Syst. Technol. 8, 288-299.

Jankovic M., Kolmanovsky I. (1998) Robust nonlinear controller for turbocharged diesel engines, American Control Conference, 1389-1394. Philadelphia, PA, USA.

Jung M. (2003) Mean-Value Modelling and Robust Control of the Airpath of a Turbocharged Diesel Engine. Department of Engineering, $P h D$ Thesis, University of Cambridge, Cambridge.

Kalman R.E. (1960) A New Approach to Linear Filtering and Prediction Problems, T. ASME-J. Basic Eng. 82 (Series D), 35-45.

Langthaler P. (2007) Model Predictive Control of a Diesel Engine Airpath. Institute for Design and Control of Mechatronical Systems, PhD Thesis, Johannes Kepler University Linz, Linz.

Ljung L. (1999) System Identification - Theory for the User, PTR Prentice Hall, Upper Saddle River, N.J.

Muske K.R., Badgwell T.A. (2002) Disturbance modeling for offsetfree linear model predictive control, J. Process Control 12, 617-632.

Ortner P., del Re L. (2007) Predictive Multivariable Control of a Diesel Engine Air Path, IEEE Trans. Control Syst. Technol. (Control Applications in Automotive Engineering) 15, 449-456.

Rückert J., Richert F., Schloßer A., Abel D., Herrmann O.E., Pfeifer A., Pischinger S. (2004) Ein Modellgestützter Prädiktiver Ansatz zur Regelung von Ladedruck und AGR-Rate beim NutzfahrzeugDieselmotor, Steuerung und Regelung von Fahrzeugen und Motoren - AUTOREG 2004.

Salcedo J.V., Pieroni E., Pérez E., Blasco X., Martínez M., García J.V. (2004) Real Time Control and Simulation of a Nonlinear model of Air Management in a Turbocharged Diesel Engine, Dept. of System Engineering and Control, Universidad Politécnica de Valencia, Valencia, Spain.

Stefanopoulou A.G., Kolmanovsky I., Freudenberg J.S. (2000) Control of variable geometry turbocharged diesel engines for reduced emissions, IEEE Trans. Control Syst. Technol. 8, 733-745.

van Nieuwstadt M.J., Kolmanovsky I.V., Moraal P.E., Stefanopoulou A., Jankovic M. (2000) EGR-VGT control schemes: experimental comparison for a high-speed diesel engine, IEEE Control Syst. Mag. 20, 63-79.

Wei X. (2006) Advanced LPV Techniques for Diesel Engines, PhD Thesis, Johannes Kepler University Linz.

Final manuscript received in January 2011 Published online in September 2011 